Laser Chem., 1999, Vol. 19, pp. 329-333

Reprints available directly from the publisher Photocopying permitted by license only
(C) 1999 OPA (Overseas Publishers Association) N.V. Published by license under the Harwood Academic Publishers imprint, part of The Gordon and Breach Publishing Group.

Printed in India.

\title{
TWO DISTINCT SOLVATED STRUCTURES OF PARA-NITROANILINE IN ACETONITRILE AND THEIR DISSOCIATION AND REASSOCIATION DYNAMICS
}

\author{
K. MOHANALINGAM ${ }^{a}$, S. YAMAGUCHI $^{\text {b }}$ \\ and H. HAMAGUCHI ${ }^{\mathrm{a}, \mathrm{c}, *}$ \\ ${ }^{a}$ Kanagawa Academy of Science and Technology, KSP East 301, \\ 3-2-1 Sakado, Takatsu, Kawasaki 213, Japan; \\ ${ }^{\mathrm{b}}$ Department of Basic Science, Graduate School of Arts and Sciences, \\ The University of Tokyo, 3-8-1 Komaba, Meguro, Tokyo 153, Japan; \\ ${ }^{\mathrm{c}}$ Department of Chemistry, School of Science, The University of Tokyo, \\ Hongo, Bunkyo, Tokyo 113, Japan
}

(Received 12 May 1997)

\begin{abstract}
We have measured the femtosecond time-resolved absorption spectra of para-nitroaniline in acetonitrile just after the photoexcitation to the lowest excited singlet state. The Singular Value Decomposition (SVD) analysis shows that two temporal evolutions follow the photoexcitation. The fast component ( $0.7 \mathrm{ps}$ time constant) describes the dissociation of the 1:2 associated form to generate the 1:0 form followed by its reassociation with AN molecules. A slower temporal evolution of $10 \mathrm{ps}$ is most likely to represent the cooling down process of the whole system.
\end{abstract}

Keywords: Femtosecond spectra; para-nitroaniline; dissociation dynamics

In previous two papers [1, 2], we showed that para-nitroaniline (pNA) exists in two distinct solvated forms in acetonitrile (AN). In the first paper, we carried out a Singular Value Decomposition (SVD) analysis of a set of ultraviolet absorption spectra of pNA in $\mathrm{AN} / \mathrm{CCl}_{4}$ mixed solvents and found that the large bathochromic shift of the pNA

\footnotetext{
${ }^{*}$ Corresponding author.
} 
absorption with increasing AN concentration is due to the change of the solvation structure of pNA. In pure $\mathrm{CCl}_{4}$, pNA exists in the free form (1:0), and in AN it exists as 1:1 and 1:2 solvated forms. In the second paper [2], we used Raman spectroscopy to elucidate the structure of these two solvated species. We concluded that in the 1:1 form, the AN molecule is attached to the amino part of pNA and that, in the 1:2 form, the second AN molecule is attached to the nitro part of pNA.

The difference in the absorption spectra of the $1: 1$ and $1: 2$ associated forms of pNA/AN (as shown in Fig. 1) makes it feasible to photoexcite selectively one of the two distinct solvated structures and examine the subsequent dissociation/solvation dynamics by ultrafast time- resolved spectroscopy. In this paper, we present the femtosecond time-resolved

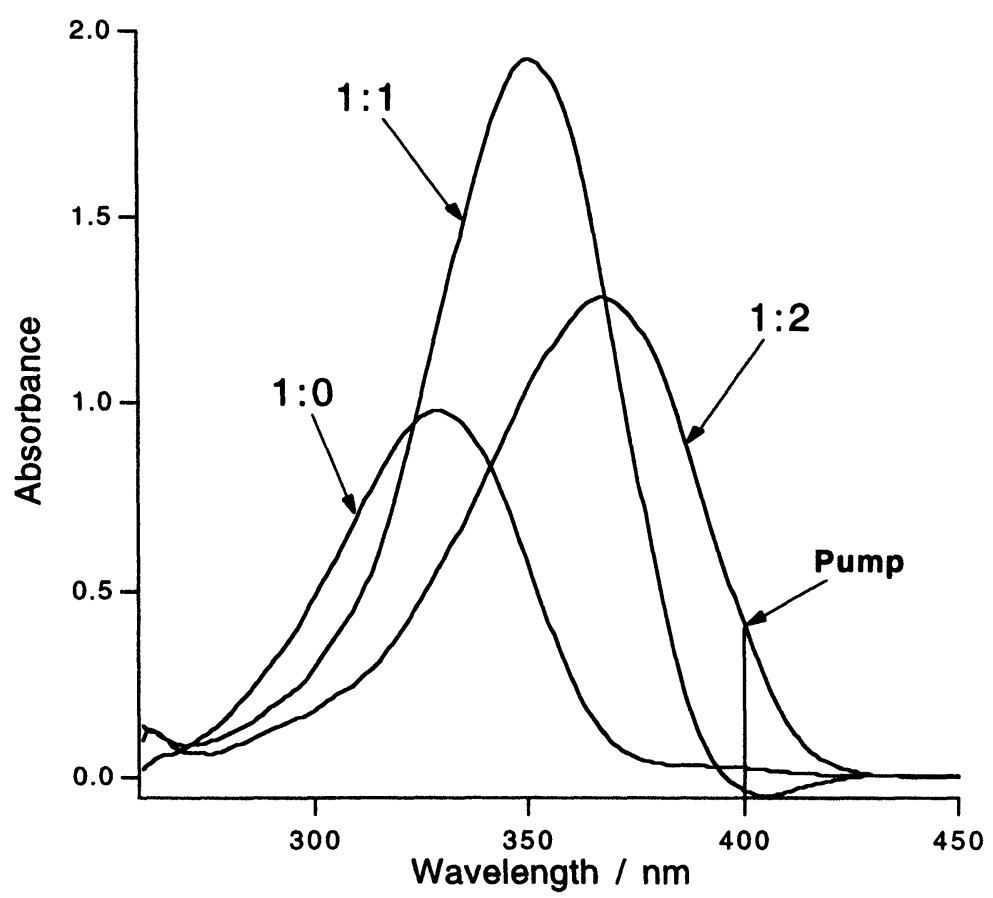

FIGURE 1 Intrinsic absorption spectra of the 1:0 (free form, in pure $\mathrm{CCl}_{4}$ ), 1:1 and 1:2 associated forms of pNA in AN. 
absorption dynamics of the solvated structures of pNA/AN following the photoexcitation at $400 \mathrm{~nm}$.

The sample of pNA was purchased from Wako Chemicals and purified by vacuum sublimation. Acetonitrile used was of HPLC grade (Wako) and used as received. The femtosecond laser system used in the present study has been described in detail earlier [3]. The ultraviolet continuum probe $(310-390 \mathrm{~nm})$ was generated by focusing femtosecond pulses $(400 \mathrm{~nm}, 300 \mathrm{fs})$ into water and was used with suitable filters. The femtosecond time-resolved absorption spectra were measured using $400 \mathrm{~nm}$ pump ( $300 \mathrm{fs}, 1 \mathrm{kHz}, 4 \mu \mathrm{J}$ ) pulses, which selectively photoexcite the $1: 2$ species.

The femtosecond time-resolved absorption spectra of pNA/AN at various time delays between the pump and probe pulses are shown in Figure 2. In addition to the broad bleaching features, the observed spectra also exhibits positive absorption at very early times. Also, it is noticeable that the bleach recovery is faster at longer wavelengths than that at shorter wavelengths. This means that more than one transient species are involved in the time-resolved spectra. In order to extract more detailed dynamical information following the photoexcitation, we have made use of an SVD analysis [5]. The analysis reveals the presence of two large singular values indicating that there are two distinct components involved. The temporal evolutions and their corresponding spectral components were obtained then by using standard curve fitting procedures [6]. The first component thus obtained represents a very fast process with a time constant of $0.7 \mathrm{ps}$, whose spectral component exhibits positive and negative absorption features (Fig. 3). The second component has a time constant of $10 \mathrm{ps}$ (not shown). In order to understand the spectral features derived from the SVD analysis, we calculated the difference absorption spectra of the 1:0 and 1:2 forms. The calculated difference spectra matches fairly well with the spectra of the fast component as shown in Figure 3. The fast component thus represents the dissociation of the 1:2 solvated structure immediately after the photoexcitation to produce the 1:0 form which then reassociates with two acetonitrile molecules to produce the 1:2 solvated form with a time constant of $0.7 \mathrm{ps}$. The slower component with a time constant of $10 \mathrm{ps}$ most probably represents the cooling down process of the whole system. 


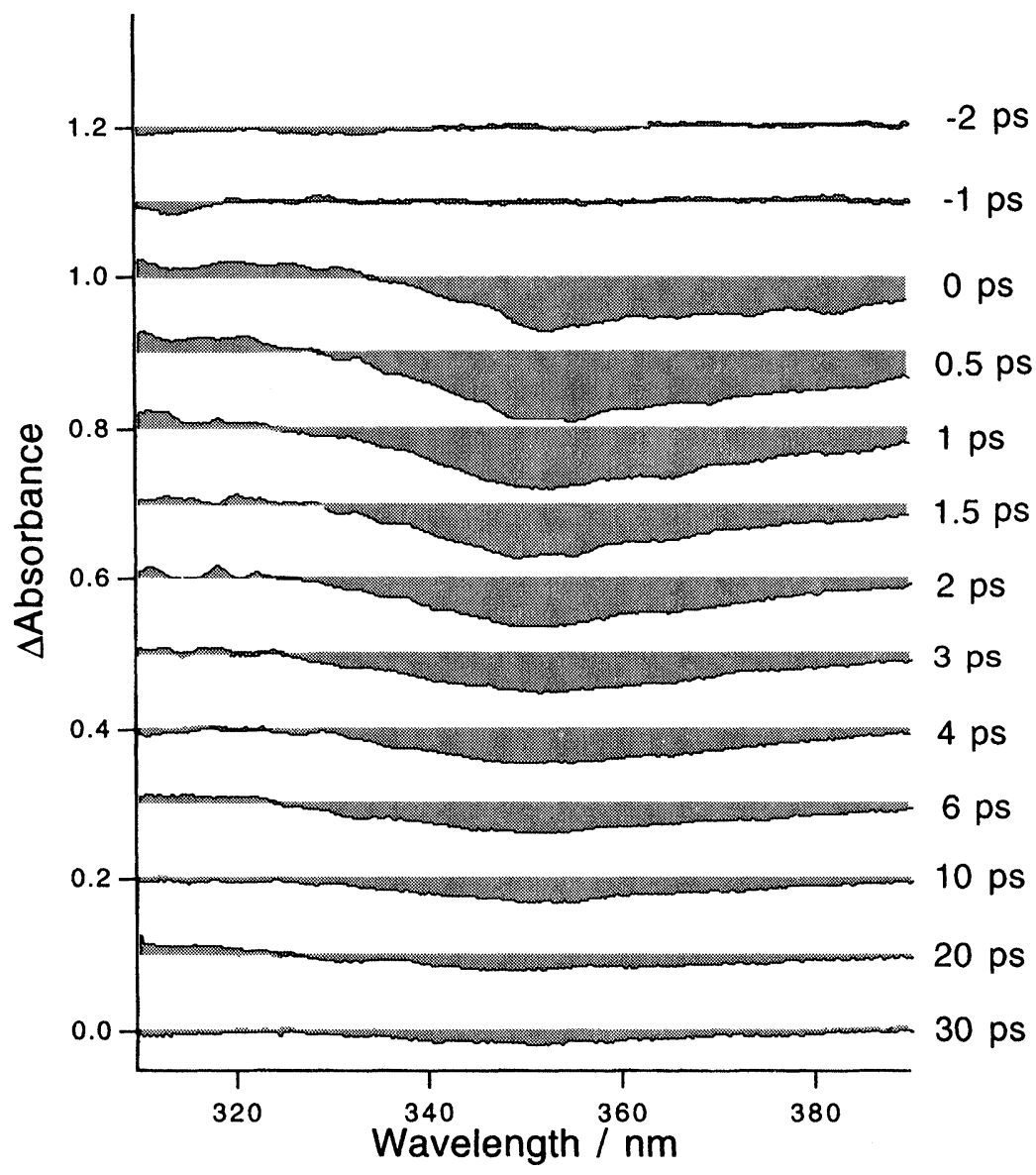

FIGURE 2 Femtosecond time-resolved absorption spectra of pNA in AN at various time delays between the pump and probe pulses.

The SVD analysis of the femtosecond time-resolved absorption spectra shows that the dissociation of the $1: 2$ form to $1: 0$ and the reassociation of the 1:0 form with AN molecule to be very fast $(0.7 \mathrm{ps})$. It seems that the dissociation is even faster than $0.7 \mathrm{ps}$ and is too fast to be observed with the present time-resolution ( $300 \mathrm{fs})$. 

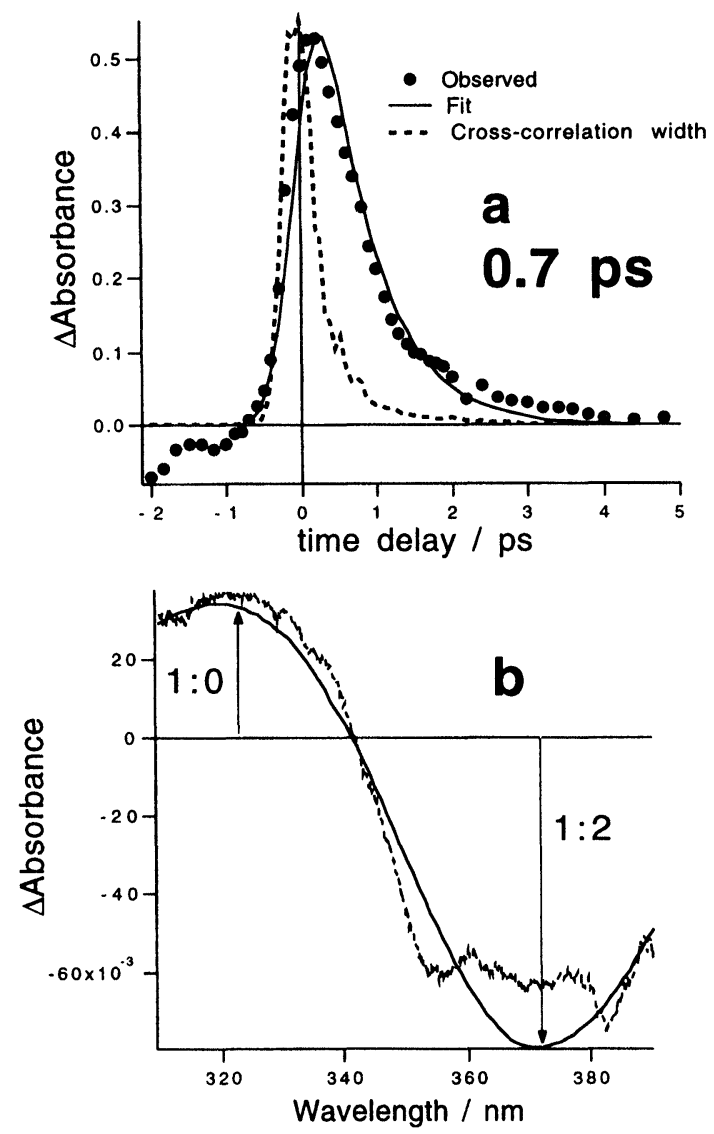

FIGURE 3 The recomposed temporal evolution (a, filled circle) and spectra of the fast component ( $b$, dotted line). The solid line in (b) is the difference spectra between the 1:0 and $1: 2$ forms (see text for details).

\section{References}

[1] Mohanalingam, K. and Hamaguchi, H. (1997). Chem. Letters, p. 157.

[2] Mohanalingam, K. and Hamaguchi, H. (1997). Chem. Letters, p. 537.

[3] Yamaguchi, S. and Hamaguchi, H. (1994). Chem. Phys. Letts., 227, 255.

[4] Yamaguchi, S. and Hamaguchi, H. (1995). Appl. Spectrosc., 49, 1513.

[5] Yuzawa, T. and Hamaguchi, H. (1995). J. Mol. Struct., 352/353, 489.

[6] Yamaguchi, S. and Hamaguchi, H. (1996). J. Mol. Struct., 379, 87. 